\title{
Molecular Subgroups of Endometrial Carcinoma in Romanian Patients
}

\author{
ANCA EVSEI ${ }^{1,4} *$, ADELINA BIRCEANU-COROBEA ${ }^{1}$, TAMÁS CSONKA ${ }^{3}$, \\ NARCIS COPCA ${ }^{2}$, MARIA SAJIN ${ }^{4,5}$ \\ ${ }^{1}$ CESITO Center, Saint Mary Clinical Hospital, Department of Pathology, 37-39 Ion Mihalache Blvd., Bucharest, Romania \\ ${ }^{2}$ CESITO Center, Saint Mary Clinical Hospital, Department of General Surgery II, 37-39 Ion Mihalache Blvd., Bucharest, \\ Romania \\ ${ }^{3}$ University of Debrecen, Faculty of Medicine, Institute of Pathology, 98 Nagyerdei Blvd., Debrecen, Hungary \\ 4 "Carol Davila" University of Medicine and Pharmacy, 8 Eroii Sanitari Blvd., Bucharest, Romania \\ ${ }^{5}$ Emergency University Hospital, Department of Pathology, 169 Splaiul Independentei, Bucharest, Romania
}

Abstract. Molecular classification of endometrial carcinoma $(E C)$ has represented a breakthrough ever since the TCGA (The Cancer Genome Atlas) project published it in 2013. Four distinct molecular subgroups were recognized based on somatic copy number alterations and tumor mutational burden: POLE (polymerase-epsilon) ultramutated, MSI (microsatellite instability) hypermutated (MSI-H), copy number low $(\mathrm{CNL})$ and copy number high $(\mathrm{CNH})$. However, implementing a standardized algorithm in current practice is far from being definitive. Our purpose in this study was to determine different molecular subgroups in a cohort of Romanian patients using cost effective and available immunohistochemistry markers and outline statistical associations with different parameters. Tissue microarrays encompassing 50 cases with previously diagnosed ECs were tested for ER, PR, HER2, p53, MSH6 and PMS2 and results showed 68\% MSI-H cases with statistical correlation with tumor size > 2 $\mathrm{cm}(p=0.028)$ and no association with overall survival. CNH ECs were reported in $26 \%$ of cases and showed important statistical significance with TILs $(p=0.041)$ and no correlations with overall survival. The CNL subgroup was reported in 74\% of ECs cases and showed statistical significance with the histopathological subtype $(p=0.006), p T$ (removal of primary tumor according to AJCC/UICC convention) ( $p=0.033)$, risk category according to ESMO (European Society of Medical Oncology) criteria $(p=0.022)$ and ER expression ( $p=0.002)$. Five-year overall survival was $84 \%$. Molecular classification is an important additional tool in current pathology guidelines for reporting ECs, but it is not currently standardized or available in all laboratories. Its importance in evolving treatment strategies for this disease is well documented so we strongly recommend routine testing for molecular prognostic factors.

Keywords: endometrial carcinoma, molecular classification, survival

\section{Introduction}

Endometrial carcinoma (EC) represents the most frequent gynecological neoplasia in developed countries, in which the incidence of cervical cancer has decreased significantly due to screening methods and prophylaxis. In Romania, however, it holds the 4th place in women malignancies after breast, cervical [1] and ovarian cancers [2]. The prevalence of EC has been on an increased trend over the years and although the age at diagnosis is usually 60-64 years, there have been numerous reports of younger female patients diagnosed with this disease.

We have come a long way as pathologists from the moment Bokhman published his epidemiological studies [3, 23] that classified endometrial carcinoma in two clinicopathological entities: type I endometrial carcinoma (low-grade endometrioid histology and association with unopposed estrogen) and type II endometrial carcinoma (unrelated to estrogen exposure and serous histology). Even now, our 
standardized guidelines for pathological reporting focus mainly on histological parameters: tumor grade, histopathological subtype, disease extension, LVSI etc. Since the moment new genetic and molecular techniques were introduced all over the world, we were offered the opportunity to study cancer biology and diversity on a deeper level.

The Cancer Genome Atlas (TCGA) endometrial collaborative project proposed in 2013 four distinct molecular subgroups of ECs: POLE (polymerase-epsilon) ultramutated, MSI (microsatellite instability) hypermutated (MSI-H), copy number low $(\mathrm{CNL})$ and copy number high $(\mathrm{CNH})$, raising the possibility for better surgical options, personalized adjuvant treatment and distinct disease surveillance [16]. This project inspired different research studies that tried to implement these ideas in an inexpensive and easy way in pathology laboratories by using different algorithms such as the ProMise algorithm [16,24].

Our purpose was to obtain a molecular insight in endometrial carcinomas in Romanian female patients, and eventually incorporate molecular prognostic factors in our routine practice.

\section{Materials and methods}

Following ethical review board approval, sample selection included 50 cases of ECs pertaining to hysterectomy specimens exclusively, that were retrieved from the Pathology Department archive from two different clinical institutions. These cases were diagnosed between 2014 and 2019. Corresponding medical files of these cases, including clinical, imaging and therapeutical parameters were obtained from the Department of Pathology from the Emergency University Hospital and Saint Mary Clinical Hospital, Bucharest, Romania.

Data collection and processing were performed using Microsoft Office Excel 2010. Hematoxylin and eosin (H\&E) stained slides for every case were reviewed and International Federation of Gynecology and Obstetrics (FIGO) revised criteria in 2018 were used for grading and staging. A representative slide for each tumor was selected and an area of the tumor was circled. Using a semiautomatic tissue microarrayer, the areas of interest of a donor block were cored using tissue punches of $1 \mathrm{~mm}$ diameter. The cores were then transferred into the recipient block. We selected three cores from each tumor and used two cores of normal endometrium for external control. Sections from these tissue microarrays were then H\&E stained and tested for spot adequacy.

Sections from the tissue microarray were stained with antibodies against ER (Ventana, catalogue number 790-4325, clone SP1, Rabbit), PR (Ventana, catalogue number 790-4296, clone 1E2, Rabbit), HER2 (Ventana, catalogue number 790-2991, clone 4B5, Rabbit), MSH6 (Ventana, catalogue number 790-5092, clone SP93, Rabbit), PMS2 (Ventana, catalogue number 790-5094, clone A16-4, Mouse), p53 (Ventana, catalogue number 790-2912, clone Bp-53-11, Mouse). Immunohistochemistry protocols enticed the following steps: deparaffinization - paraffin sections of 4- $\mu \mathrm{m}$ thickness were baked overnight at $50^{\circ} \mathrm{C}$. The deparaffinization step was done in the Ventana Discovery XT platform using EZ prep solution (an aqueous-based detergent solution with $0.5 \%$ ProClin 300 as a preservative). The standard antigen retrieval method was Heat Induced Epitope Retrieval (HIER) in Tris-EDTA (TrisEthylenediaminetetraacetic acid) buffer $p \mathrm{H} 7.8$ at $95^{\circ} \mathrm{C}$ for $44 \mathrm{~min}$ (standard $\mathrm{CC} 1$ - standard conditioning solution) and was performed in the Ventana Discovery XT. If expected results were not achieved, we tested a different time or used Citrate-based buffer $p \mathrm{H} 6.0$ or Protease antigen retrieval instead. For blocking endogenous peroxides and protein, these steps were done in the Ventana Discovery XT platform using Inhibitor CM (ChromoMap). Staining protocol for Ventana Discovery XT can be resumed as following: 1 -Tissue Sample, Paraffin; 2-Deparaffinization in EZ prep $75^{\circ} \mathrm{C} 8$ min; 3-Cell Conditioning using Conditioner \#1, Standard $\mathrm{CC} 1,95^{\circ} \mathrm{C} 44 \mathrm{~min}$; 4-Block with Inhibitor $\mathrm{CM}, 37^{\circ} \mathrm{C} 4$ minutes; 5-No Heat; 6-Incubation with primary antibody for $60 \mathrm{~min}$; 7-Apply one drop of OmniMap anti-Rb HRP and incubate for $16 \mathrm{~min} / A p p l y$ one drop of OmniMap anti-Ms HRP and incubate for 16 min; 8-Apply one drop of DAB CM and One Drop $\mathrm{H}_{2} \mathrm{O}_{2} \mathrm{CM}$, incubate $8 \mathrm{~min}$; 9-Apply one drop of Copper CM, incubate 5min; 10-Counterstain with Hematoxylin, incubate for $8 \mathrm{~min}$; 11-Post counterstain with Bluing Reagent, incubate for 8 min; 12-Slide Cleaning; 13-Wash the slides in warm tap water with 
detergent and dehydrate in graded ethanol and xylene; 14-Coverslip the slides in permanent mounting media. Technicians followed the staining protocols according to the manufacturer's instructions.

ER and PR status were determined according to standardized evaluation guidelines into positive and negative, taking into consideration proper external and internal control. HER2 was evaluated in the following way: negative (score 0, score 1+), equivocal (score 2+) and positive (score 3+). MSH6 and PMS2 were scored according to intact/loss of nuclear expression in tumor cells. Subsequently, we selected cases for the MSI-H category (MSH6 and/or PMS2 loss of nuclear expression). P53 staining facilitated the following molecular subgroups: $\mathrm{CNH}$ category (abnormal strong diffuse p53 overexpression $>90 \%$ or abnormal null expression - complete loss of p53 expression) and CNL (normal expression - p53 wild type).

Statistical analysis used the chi-square test for categorical and binary variables. Independent Sample T-test was used for numerical and categorical variables. Sample proportion test was used for binary variables and Kaplan-Meier survival curves for overall survival.

\section{Results and discussions}

Patient's ages ranged from 39 to 75 years with a mean age of 63 years. According to the most recent studies in Romania [2], the maximum incidence is situated between 60 and 64 years old, the mortality rate of the women under 65 years old being high. Tumor diagnosis was performed exclusively on surgical specimens. The most frequent age category was 61-70, which consists with most studies, with $12 \%$ of patients presenting omentectomies with tumor involvement, $8 \%$ with positive peritoneal cytology and $8 \%$ of cases with preoperative atypical/malignant pap smears. Brincat et al. [3] revealed in an evidence-based study that apart from the macroscopic assessment of the omentum during surgical procedures, which is highly advisable, total omentectomy with subsequent histological analysis is far superior regarding tumor spread and has shown improved overall survival.

Although new staging guidelines do not include positive peritoneal cytology as a parameter and prognosis remains uncertain, one recent study [4] performed on a large cohort of patients proved that positive peritoneal cytology was associated with decreased overall survival for women with FIGO I/II stage, including those with low-grade endometrioid endometrial carcinoma. $64 \%$ of our cases showed tumor size $>2 \mathrm{~cm}$ with $14 \%$ associated lymph node metastasis and $5 \%$ of cases with previous histopathological diagnosis of endometrial hyperplasia without or with atypia. Kemi et al. [5] showed in one study that tumor dimension and lower uterine involvement are significantly associated with lymph node metastasis and advanced stage disease at the time of comprehensive surgical staging.

Histological subtyping, followed by confirmatory immunohistochemistry revealed 41 endometrioid carcinomas, 4 serous carcinomas, 2 clear cell carcinomas, 2 mixed carcinomas and 1 carcinosarcoma. This tumor subtype distribution is consistent with another research [2].

FIGO grade II was the most frequent (42\%); $78 \%$ of cases showed lympho-vascular invasion (LVSI); tumor necrosis was present in 74\% of cases; adenomyosis was present in 32 cases, 2 of them with tumor involvement. 56\% of cases presented with moderate TILs (tumor-infiltrating lymphocytes). Vermij et al. $[7,8]$ proved that LVSI in EC patients is connected with an increased risk of lymph node metastasis, locoregional as well as distant recurrences, regardless of stage and histotype. For that matter, LVSI will remain essential in risk stratification schemes, escalating adjuvant treatment regimens in those patients with substantial LVSI. Tumor necrosis was established as a parameter for tumor aggressiveness in numerous studies and has been associated with poor overall survival. One study [9] explained its presence through increased tissue hypoxia, angiogenesis and inflammation in tumor microenvironment. ER negative tumors were frequently associated with this parameter, same as in our study.

Myometrium invasion was more than one-half in 31 cases. $62 \%$ of patients showed diffusely infiltrative pattern of invasion and only one patient had MELF pattern of invasion. Deep myometrial invasion is highly correlated with tumor size, lymph node involvement and distant metastasis [10]. Tumors with diffusely infiltrative patterns of invasion are the most frequent and this is in agreement with other results [11]. However, MELF pattern, which has been linked with myometrial invasion, LVSI and 
lymph node involvement [11-13] and is currently considered a significant prognostic pattern, was represented in our study in only one patient.

After revising oncological and clinical patient data, we found that only five patients received neoadjuvant chemotherapy or radiotherapy. Final TNM staging according to new pathological guidelines revealed that the most frequent FIGO stage was FIGO IB (24\%). Following ESMO criteria, we found that the most common group was the High-Risk Intermediate Group with 15 confirmed cases. ESMO criteria and FIGO stage in our group correlate significantly with lymph node removal and staging, which is in agreement with current guidelines [14].

Our study group was divided in 3 molecular categories using p53, MSH6 and PMS2 testing. Results identified 34 cases as MSI-H, 13 cases as CNH and 37 cases as CNL. Extensive descriptive statistics on distribution between demographic, clinical and pathologic parameters and the three molecular subgroups are shown below (Table 1).

Table 1. Descriptive statistics of cohort $(n=50)$, demographic, clinicopathological and molecular parameters within the three molecular subgroups.

\begin{tabular}{|c|c|c|c|c|}
\hline & Total & MSI-H & $\mathrm{CNH}$ & $\mathrm{CNL}$ \\
\hline \multirow{4}{*}{$\begin{array}{l}\text { Age } \\
\text { Mean }( \pm \text { SD) } \\
\text { Median (IQR) } \\
\text { Absent }\end{array}$} & & & & \\
\hline & $62.82( \pm 8.108)$ & $59.38( \pm 7.957)$ & $62.92( \pm 9.802)$ & $63.03( \pm 7.429)$ \\
\hline & $63(11)$ & $\begin{array}{l}59(11) \\
\end{array}$ & $65(13)$ & $63(9)$ \\
\hline & 0 & 0 & 0 & 0 \\
\hline \multirow{4}{*}{$\begin{array}{l}\text { Omentectomy } \\
\text { Present - tumor involvement } \\
\text { Present - no tumor } \\
\text { involvement } \\
\text { Absent } \\
\end{array}$} & & & & \\
\hline & $6(50 \%)$ & $1(16.7 \%)$ & $1(16.7 \%)$ & $5(83.3 \%)$ \\
\hline & $6(50 \%)$ & $0(0 \%)$ & $3(50 \%)$ & $2(33.3 \%)$ \\
\hline & 38 & 0 & 0 & 0 \\
\hline \multirow{4}{*}{$\begin{array}{l}\text { Peritoneal cytology } \\
\text { Positive } \\
\text { Negative } \\
\text { Absent }\end{array}$} & & & & \\
\hline & $3(37.5 \%)$ & $0(0 \%)$ & $2(66.7 \%)$ & $0(0 \%)$ \\
\hline & $5(62.5 \%)$ & $3(60 \%)$ & $3(60 \%)$ & $3(60 \%)$ \\
\hline & 42 & 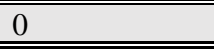 & 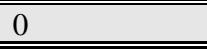 & 0 \\
\hline \multirow{4}{*}{$\begin{array}{l}\text { Pap smear } \\
\text { Reactive } \\
\text { Positive } \\
\text { Absent }\end{array}$} & & & & \\
\hline & $6(60 \%)$ & $1(16.7 \%)$ & $1(16.7 \%)$ & $6(100 \%)$ \\
\hline & $4(40 \%)$ & $1(25 \%)$ & $1(25 \%)$ & $3(75 \%)$ \\
\hline & 40 & 0 & 0 & 0 \\
\hline \multirow{4}{*}{$\begin{array}{l}\text { Tumor dimension } \\
<2 \mathrm{~cm} \\
>2 \mathrm{~cm} \\
\text { Absent }\end{array}$} & & & & \\
\hline & $17(34 \%)$ & $2(11.8 \%)$ & $5(29.4 \%)$ & $12(70.6 \%)$ \\
\hline & $33(66 \%)$ & $14(42.4 \%)$ & $8(24.2 \%)$ & $27(81.8 \%)$ \\
\hline & 0 & 0 & 0 & 0 \\
\hline \multicolumn{5}{|l|}{ Preneoplastic lesions } \\
\hline \multirow{3}{*}{$\begin{array}{l}\text { HWA } \\
\text { AH/EIN } \\
\text { Absent }\end{array}$} & $1(20 \%)$ & $1(100 \%)$ & $0(0 \%)$ & $1(100 \%)$ \\
\hline & $4(80 \%)$ & $1(25 \%)$ & $1(25 \%)$ & $3(75 \%)$ \\
\hline & 45 & $\begin{array}{l}0 \\
\end{array}$ & $\begin{array}{l}0 \\
\end{array}$ & $\begin{array}{ll}0 \\
\end{array}$ \\
\hline \multirow{7}{*}{$\begin{array}{l}\text { Histopathological } \\
\text { Endometrioid } \\
\text { Serous } \\
\text { Clear cell } \\
\text { Mixed } \\
\text { Carcinosarcoma } \\
\text { Absent }\end{array}$} & & & & \\
\hline & $41(82 \%)$ & $15(36.6 \%)$ & $9(22 \%)$ & $35(85.4 \%)$ \\
\hline & $4(8 \%)$ & $0(0 \%)$ & $2(50 \%)$ & $2(50 \%)$ \\
\hline & $2(4 \%)$ & $0(0 \%)$ & $1(50 \%)$ & $0(0 \%)$ \\
\hline & $2(4 \%)$ & $1(50 \%)$ & $0(0 \%)$ & $2(100 \%)$ \\
\hline & $1(2 \%)$ & $0(0 \%)$ & $1(100 \%)$ & $0(0 \%)$ \\
\hline & 0 & 0 & 0 & 0 \\
\hline \multicolumn{5}{|l|}{ FIGO Grade } \\
\hline \multirow{4}{*}{$\begin{array}{l}\text { Grade } 1 \\
\text { Grade } 2 \\
\text { Grade } 3 \\
\text { Absent }\end{array}$} & $9(18 \%)$ & $2(22 \%)$ & $3(33 \%)$ & $7(77.8 \%)$ \\
\hline & $21(42 \%)$ & $6(28.6 \%)$ & $3(14.3 \%)$ & $19(90.5 \%)$ \\
\hline & $20(40 \%)$ & $8(40 \%)$ & $7(35 \%)$ & $13(65 \%)$ \\
\hline & 0 & 0 & 0 & 0 \\
\hline FIGO stage & & & & \\
\hline
\end{tabular}




\section{Revista de Chimie}

https://revistadechimie.ro

https://doi.org/10.37358/Rev.Chim.1949

\begin{tabular}{|c|c|c|c|c|}
\hline \multirow{8}{*}{$\begin{array}{l}\text { IA } \\
\text { IB } \\
\text { II } \\
\text { IIIA } \\
\text { IIIB } \\
\text { IIIC1 } \\
\text { IIIC2 } \\
\text { Absent }\end{array}$} & $9(18 \%)$ & $3(33 \%)$ & $2(22 \%)$ & $9(100 \%)$ \\
\hline & $12(24 \%)$ & $3(25 \%)$ & $4(33.3 \%)$ & $9(75 \%)$ \\
\hline & $10(20 \%)$ & $4(40 \%)$ & $101(10 \%)$ & $9(90 \%)$ \\
\hline & $7(14 \%)$ & $2(28.6 \%)$ & $4(57.1 \%)$ & $3(42.9 \%)$ \\
\hline & $5(10 \%)$ & $3(60 \%)$ & $0(0 \%)$ & $4(80 \%)$ \\
\hline & $3(65)$ & $1(33 \%)$ & $1(33 \%)$ & $2(66.7 \%)$ \\
\hline & $4(8 \%)$ & 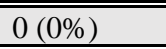 & $10(25 \%)$ & $3(75 \%)$ \\
\hline & 0 & 0 & 0 & 0 \\
\hline \multirow{7}{*}{$\begin{array}{l}\text { T } \\
\text { T1a } \\
\text { T1b } \\
\text { T2 } \\
\text { T3a } \\
\text { T3b } \\
\text { Absent }\end{array}$} & & & & \\
\hline & $9(18 \%)$ & $3(33 \%)$ & $2(22 \%)$ & $9(100 \%)$ \\
\hline & $13(26 \%)$ & $3(23.1 \%)$ & $4(30.8 \%)$ & $10(76.9 \%)$ \\
\hline & $11(22 \%)$ & $4(36.4 \%)$ & $1(9.1 \%)$ & $10(90.9 \%)$ \\
\hline & $11(22 \%)$ & $3(27.3 \%)$ & $5(45.5 \%)$ & $5(45.5 \%)$ \\
\hline & $6(12 \%)$ & $3(50 \%)$ & $1(16.7 \%)$ & $5(83.3 \%)$ \\
\hline & 0 & 0 & 0 & 0 \\
\hline \multirow{5}{*}{$\begin{array}{l}\mathrm{N} \\
\text { N0 } \\
\text { N1 } \\
\text { N2 } \\
\text { Absent }\end{array}$} & & & & \\
\hline & $32(64 \%)$ & $12(37.5 \%)$ & $8(25 \%)$ & $27(84.4 \%)$ \\
\hline & $6(12 \%)$ & $3(50 \%)$ & $2(33.3 \%)$ & $4(66.7 \%)$ \\
\hline & $1(2 \%)$ & $0(0 \%)$ & $0(0 \%)$ & $1(100 \%)$ \\
\hline & 0 & 0 & 0 & 0 \\
\hline \multirow{4}{*}{$\begin{array}{l}\text { M } \\
\text { M1 } \\
\text { M0 } \\
\text { Absent }\end{array}$} & & & & \\
\hline & $46(92 \%)$ & $16(34.8 \%)$ & $11(23.9 \%)$ & $37(80.4 \%)$ \\
\hline & $4(8 \%)$ & $0(0 \%)$ & $2(50 \%)$ & $2(50 \%)$ \\
\hline & 0 & 0 & 0 & 0 \\
\hline \multirow{5}{*}{$\begin{array}{l}\text { Adenomyosis } \\
\text { No adenomyosis } \\
\text { Adenomyosis with tumor } \\
\text { involvement } \\
\text { Adenomyosis with no tumor } \\
\text { involvement } \\
\text { Absent } \\
\end{array}$} & & & & \\
\hline & $32(64 \%)$ & $10(31.2 \%)$ & $9(28.1 \%)$ & $24(75 \%)$ \\
\hline & $2(4 \%)$ & $0(0 \%)$ & $0(0 \%)$ & $2(100 \%)$ \\
\hline & $16(32 \%)$ & $6(37.5 \%)$ & $4(25 \%)$ & $13(81.2 \%)$ \\
\hline & 0 & 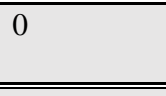 & $\overline{00}$ & $\overline{00}$ \\
\hline \multirow{4}{*}{$\begin{array}{l}\text { LVSI } \\
\text { Yes } \\
\text { No } \\
\text { Absent } \\
\end{array}$} & & & & \\
\hline & $39(78 \%)$ & $14(35.9 \%)$ & $11(18.2 \%)$ & $29(74.4 \%)$ \\
\hline & $11(22 \%)$ & $2(18.2 \%)$ & $2(18.2 \%)$ & $10(90.9 \%)$ \\
\hline & 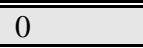 & 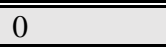 & 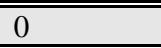 & 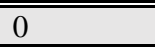 \\
\hline \multirow{4}{*}{$\begin{array}{l}\text { Tumor } \mathrm{n} \\
\text { Yes } \\
\text { No } \\
\text { Absent }\end{array}$} & & & & \\
\hline & $37(74 \%)$ & $12(32.4 \%)$ & $12(32.4)$ & $27(73 \%)$ \\
\hline & $13(26 \%)$ & $4(30.8 \%)$ & $\begin{array}{l}1(7.7 \%) \\
\end{array}$ & $12(92.3 \%)$ \\
\hline & 0 & 0 & 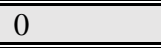 & 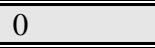 \\
\hline \multicolumn{5}{|l|}{ "TILs } \\
\hline \multirow{4}{*}{$\begin{array}{l}\text { Minimum } \\
\text { Moderate } \\
\text { Marked } \\
\text { Absent }\end{array}$} & $14(28 \%)$ & $2(14.3 \%)$ & $2(14.3 \%)$ & $12(85.7 \%)$ \\
\hline & $28(56 \%)$ & $9(32.1 \%)$ & $11(39.3 \%)$ & $9(67.9 \%)$ \\
\hline & $8(16 \%)$ & $5(62.5 \%)$ & $0(0 \%)$ & $8(100 \%)$ \\
\hline & $\begin{array}{l}0 \\
\end{array}$ & $\begin{array}{l}0 \\
\end{array}$ & $\begin{array}{l}0 \\
\end{array}$ & 0 \\
\hline \multirow{5}{*}{$\begin{array}{l}\text { Myometrial invasion } \\
<50 \% \\
>50 \% \\
\text { No myometrial invasion } \\
\text { Absent }\end{array}$} & & & & \\
\hline & $18(36 \%)$ & $6(33.3 \%)$ & $5(27.8 \%)$ & $15(83.3 \%)$ \\
\hline & $31(62 \%)$ & $10(32.3 \%)$ & $7(22.6 \%)$ & $\begin{array}{l}24(77.4 \%) \\
\end{array}$ \\
\hline & $1(2 \%)$ & $0(0 \%)$ & $1(100 \%)$ & 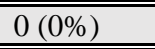 \\
\hline & 0 & 0 & 0 & 0 \\
\hline \multirow{6}{*}{$\begin{array}{l}\text { Pattern of invasion } \\
\text { Pushing } \\
\text { Diffusely Infiltrative } \\
\text { Adenomyosis } \\
\text { MELF } \\
\text { Absent }\end{array}$} & & & & \\
\hline & $16(32 \%)$ & $2(12.5 \%)$ & $2(12.5 \%)$ & $15(93.8 \%)$ \\
\hline & $31(62 \%)$ & $13(41.9 \%)$ & $11(35.5 \%)$ & $\begin{array}{l}21(67.7 \%) \\
\end{array}$ \\
\hline & $2(4 \%)$ & $0(0 \%)$ & $0(0 \%)$ & $2(100 \%)$ \\
\hline & $1(2 \%)$ & $1(100 \%)$ & $0(0 \%)$ & $1(100 \%)$ \\
\hline & 0 & 0 & 0 & 0 \\
\hline
\end{tabular}




\begin{tabular}{|c|c|c|c|c|}
\hline \multirow{5}{*}{$\begin{array}{l}\text { Treatment } \\
\text { Chemotherapy } \\
\text { Radiotherapy } \\
\text { No treatment } \\
\text { Absent }\end{array}$} & & & & \\
\hline & $4 \quad 4(8 \%)$ & $101(25 \%)$ & $2(50 \%)$ & $3(75 \%)$ \\
\hline & $1(2 \%)$ & $0(0 \%)$ & $0(0 \%)$ & $10(100 \%)$ \\
\hline & $404(90 \%)$ & $15(33.3 \%)$ & $11(24.4 \%)$ & $35(77.8 \%)$ \\
\hline & 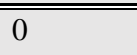 & $\overline{00}$ & 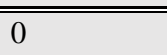 & 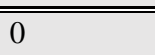 \\
\hline \multirow{7}{*}{$\begin{array}{l}\text { ESMO } \\
\text { Low } \\
\text { Intermediate } \\
\text { High Intermediate } \\
\text { High } \\
\text { Advanced } \\
\text { Absent }\end{array}$} & & & & \\
\hline & $3(6 \%)$ & $1(33.3 \%)$ & $1(33.3 \%)$ & $3(100 \%)$ \\
\hline & $4(8 \%)$ & "1(25\%) & "1(25\%) & $3(75 \%)$ \\
\hline & $15(30 \%)$ & $6(40 \%)$ & $2(13.3 \%)$ & $15(100 \%)$ \\
\hline & $14(28 \%)$ & $4(28.6 \%)$ & $3(21.4 \%)$ & $11(78.6 \%)$ \\
\hline & $14(28 \%)$ & $4(28.6 \%)$ & $6(42.9 \%)$ & $7(50 \%)$ \\
\hline & 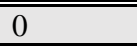 & 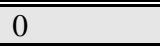 & 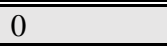 & 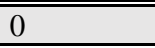 \\
\hline \multirow{4}{*}{$\begin{array}{l}\text { ER } \\
\text { Positive } \\
\text { Negative } \\
\text { Absent }\end{array}$} & & & & \\
\hline & $43(86 \%)$ & $15(34.9 \%)$ & $9(20.9 \%)$ & $37(86 \%)$ \\
\hline & $7(14 \%)$ & $1(14.3 \%)$ & $4(57.1 \%)$ & $2(28.6 \%)$ \\
\hline & 0 & 0 & 0 & 0 \\
\hline \multirow{4}{*}{$\begin{array}{l}\text { PR } \\
\text { Positive } \\
\text { Negative } \\
\text { Absent }\end{array}$} & & & & \\
\hline & $42(84 \%)$ & $14(33.3 \%)$ & $10(23.8 \%)$ & $35(83.3 \%)$ \\
\hline & $8(16 \%)$ & $2(25 \%)$ & $3(37.5 \%)$ & $4(50 \%)$ \\
\hline & 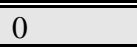 & 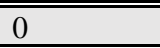 & 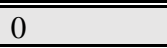 & 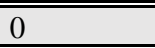 \\
\hline \multirow{4}{*}{$\begin{array}{l}\text { HER2 } \\
\text { Negative } \\
\text { Positive } \\
\text { Absent }\end{array}$} & & & & \\
\hline & $499(98 \%)$ & $1016(32.7 \%)$ & $12(24.5 \%)$ & $39(79.6 \%)$ \\
\hline & $101(2 \%)$ & 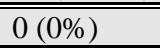 & $10(100 \%)$ & $0(0 \%)$ \\
\hline & $\begin{array}{l}0 \\
\end{array}$ & $\begin{array}{l}0 \\
\end{array}$ & 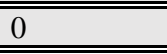 & 0 \\
\hline
\end{tabular}

HWA=Hyperplasia without Atypia; AH/EIN= Atypical Hyperplasia /Endometrial Intraepithelial Neoplasia.

MSI-H endometrial tumors were identified most frequently in our study in younger patients (median age $=59$ years) with: endometrioid and mixed histological subtypes, FIGO grade 3, FIGO stage II, absent lymph node involvement, $34 \%$ cases with distant metastasis, adenomyosis without tumor involvement, present LVSI and tumor necrosis, moderate TILs and diffusely infiltrative pattern. High-Risk Intermediate group was the most frequent category. ER and PR expression were positive in almost all cases and HER2 was negative in all cases. Statistical analysis showed prognostic significance between the MSI-H group and tumor dimension $>2 \mathrm{~cm}$ and pattern of invasion $(\mathrm{p}=0.028)$. Overall survival was not correlated with this group in our study.

According to recent studies, MSI-H/hypermutated tumors which include Lynch syndrome cancers and are represented by loss of MMR (Mismatch Repair) through hypermethylation of one of MMR proteins, comprise $25-30 \%$ of endometrial carcinomas [15]. Our study revealed a significant percentage of MSI-H tumors (68\%) diagnosed by evaluating loss of MSH6 and/or PMS2 expression, which contradicts various research performed on much larger cohorts $[8,15,16]$. Other parameters of our study correlated with this molecular subgroup similarly with other conducted studies: higher-grade ECs, endometrioid-type histology, moderate/marked TILs, substantial LVSI [8].

CNH endometrial tumors were identified most frequently in our study in patients with: omentectomies with tumor involvement, positive peritoneal cytology, endometrioid ( 9 cases) and serous histopathological subtypes, higher tumor grade, FIGO stage IIIA, present LVSI, similar percentage of cases with tumor necrosis as the MSI-H group, Advanced Risk group, infrequent ER and PR positivity and HER2 positivity (HER2 3+) in one case. Statistical analysis showed important prognostic significance with TILs $(\mathrm{p}=0.041)$ and no correlations with overall survival.

$\mathrm{CNH}$ (serous-like tumors) comprise $26 \%$ of endometrial carcinoma, same as in our study. These tumors have low mutational frequency and are usually defined by serous histology, although numerous reports have highlighted high-grade endometrioid tumors (35\%) that fall in this cluster [15]. In our cohort, we identified $22 \%$ of high-grade endometrioid tumors, which expressed an abnormal pattern of 
p53 staining. Other parameters coincide with other studies regarding frequency: higher-grade ECs, high FIGO stage, substantial LVSI, high tumor necrosis, high capacity to spread in the abdominal cavity. Some studies identified expression of HER 2 in $25 \%$ of serous or serous-like tumors [17], which explains at least partially, why the only HER2 positive case in our case study belonged to this molecular subgroup. This molecular subgroup has been frequently associated with poor disease-survival and overall survival [18].

CNL endometrial tumors showed frequency in patients with: omentectomies with tumor involvement, positive pap smears, tumor dimension $>2 \mathrm{~cm}$, preneoplastic lesions such as endometrial hyperplasia with atypia, endometrioid and mixed histopathological subtypes, FIGO grade 2 (90,5\% cases), FIGO stages I and II, the highest percentage of lymph node involvement $(66.7 \%)$ and distant metastasis (37 cases), the only 2 cases with adenomyosis with tumor involvement, frequent LVSI, highest percentage of tumor necrosis, minimum TILs, MELF pattern of invasion, neoadjuvant treatment, High Risk group. ER and PR positivity were the highest in this subgroup and HER2 was negative. Statistical analysis showed prognostic significance between histopathological subtype and CNL group $(\mathrm{p}=0.06)$, pT (removal of primary tumor according to AJCC/UICC convention) and CNL group $(\mathrm{p}=0.033)$, risk category according to ESMO criteria and CNL group $(\mathrm{p}=0.022), \mathrm{ER}$ expression and CNL group ( $\mathrm{p}=0.002)$.

This particular molecular subgroup has no specific molecular incriminated driver [19]. Although this largely represented subclass is defined by a low mutational burden, there are numerous studies that show that these have an aggressive clinical behavior $[8,18]$. Studies show a $65 \%$ percentage of this group, but our cohort shows a much higher percentage (74\%). In addition, we noticed that this subgroup is linked to the highest lymph node involvement and the highest percentage of distant metastasis, which is consistent with another research [20]. Statistical significance with the histopathological type, which is usually endometrioid and ER positivity offers additional information for treatment as this subgroup is the most difficult to manage clinically and therapeutically [21].

Five-year overall survival was $84 \%$. Kaplan-Meier survival curves showed prognostic significance with omentectomy $(\mathrm{p}=0.044)$, histopathological subtype $(\mathrm{p}=0.002)$, myometrial invasion $(\mathrm{p}=0.001), \mathrm{ER}$ expression $(\mathrm{p}=0.006)$ and PR expression $(\mathrm{p}=0.001)$. These results are highly consistent with other studies and reveal that an endometrioid histology, $<1 / 2$ myometrial invasion, ER and PR positivity correlate with good overall survival and good prognosis [22].

Our study performed on 50 cases of endometrial carcinomas diagnosed in Romanian patients revealed different molecular patterns than in other larger cohorts. We found a much larger percentage of MSI-H endometrial carcinomas (68\%) which should represent a red flag and prompt further investigation to exclude genetic syndromes (Lynch syndrome), since endometrial carcinoma is often considered a `sentinel cancer' and patients often have other digestive malignancies. CNL endometrial carcinomas were also higher in percentage in our group (74\%) outlining the fact that Romanian female patients are susceptible to aggressive tumors with high lymph node involvement and higher percentages of distant metastasis, which implies a poor response to combined therapy and a lower overall survival.

This research, however, is subject to several limitations. Sample size of 50 cases of endometrial carcinomas in relation to the entire population of female patients with this diagnosis in Romania is considered small, but future work will include larger cohorts from different geographical areas in order to contribute to the emerging global picture of this disease.

\section{Conclusions}

In conclusion, we found a much larger percentage of MSI-H endometrial carcinomas (68\%) which should represent a red flag and prompt further investigation to exclude genetic syndromes (Lynch syndrome), since endometrial carcinoma is often considered a `sentinel cancer` and patients often have other digestive malignancies. CNL endometrial carcinomas were also higher in percentage in our group (74\%) outlining the fact that Romanian female patients are susceptible to aggressive tumors with high 
lymph node involvement and higher percentages of distant metastasis, which implies a poor response to combined therapy and a lower overall survival.

Endometrial carcinoma has seen an interesting evolution from a purely histological-based classification to a complex incorporation of gene sequencing and genomic analysis. Its increasingly recognition as a biologically diverse tumor has determined an evolving quest in finding molecular prognostic factors, easily tested and widely available. Implementing a molecular strategy in routine practice will eventually guide surgery, adjuvant treatment, disease surveillance, fertility sparing treatments etc. and identification of surrogate markers that accurately reflect molecular subtypes is the only feasible way to determine that outcome.

\section{References}

1. TATARU, A.L., FURAU, G., AFILON, J., IONESCU, C., DIMITRIU, M., BRATU, O.G., TIT, D.M., BUNGAU, S., FURAU, C. The situation of cervical cancers in the context of female genital cancer clustering and burden of disease in Arad County, Romania, J. Clin. Med. 8(1), 2019, E96; https://doi.org/10.3390/jcm8010096.

2. BOHÎLȚEA, R. E. et al., Evaluation of endometrial cancer epidemiology in Romania, Journal of Medicine and Life, 8(2), 2015, 218-25.

3. BRINCAT, M.R., BARON, Y.M, BORG, E., Omentectomy in endometrial cancer: an evidence-based insight, European Journal of Gynaecological Oncology, 28(4), 2017.

4. SEAGLE, B-LL., ALEXANDER, AL., LANTSMAN, T., et al., Prognosis and treatment of positive peritoneal cytology in early endometrial cancer: matched cohort analyses from the National Cancer Database, Am J Obstet Gynecol, 218:329, 2018, 1-15.

5. DOLL, K. M., DENSLOW, S., TSENG, J., GEHRIG, P.A., FADER, A.N., High-grade endometrial cancer: Revisiting tumor size and the lower uterine segment, Journal of Clinical Oncology, 31(15), 2013, 5589-5589.

6. KARNEZIS, A.N., LEUNG, J., MCCONECHY, M.K., YANG, W., CHOW, C., KOBEL, M., LEE, C-H., HUNTSMAN, D.G., TALHOUK, A., Evaluation of endometrial carcinoma prognostic immunohistochemistry markers in the context of molecular classification, J Path Clin Res, 3, 2017, 279-293.

7. SOSLOW, R.A., TORNOS, C., PARK, K.J., et al., Endometrial Carcinoma Diagnosis: Use of FIGO Grading and Genomic Subcategories in Clinical Practice: Recommendations of the International Society of Gynecological Pathologists, Int J Gynecol Pathol, 38, 2019, 64-74.

8. VERMIJ, L., SMIT, V., NOUT, R., BOSSE, T., Incorporation of molecular characteristics into endometrial cancer management, Histopathology, 76, 2020, 52-63.

9. BREDHOLT, G., MANNELQVIST, M., STEFANSSON, I.M., BIRKELAND, E., et al., Tumor necrosis is an important hallmark of aggressive endometrial cancer and associates with hypoxia, angiogenesis and inflammation responses, Oncotarget, 6(37), 2015, 39676-91.

10. NAKAMURA, K., NAKAYAMA, K., ISHIKAWA, N., et al., Preoperative tumor size is associated with deep myometrial invasion and lymph node metastases and is a negative prognostic indicator for patients with endometrial carcinoma, Oncotarget, 9(33), 2018, 23164-23172.

11. RUZ-CARACUEL, I., RAMÓN-PATINO, J.L., LÓPEZ-JANEIRO, Á., YÉBENES, L., et al., Myoinvasive Pattern as a Prognostic Marker in Low-Grade, Early-Stage Endometrioid Endometrial Carcinoma, Cancers, 11, 2019, 1845.

12. PRODROMIDOU, A., VORGIAS, BAKOGIANNIS, K., et al., MELF pattern of myometrial invasion and role in possible endometrial cancer diagnostic pathway: A systematic review of the literature, Eur J Obstet Gynecol Reprod Biol, 230, 2018, 147-152.

13. JOEHLIN-PRICE, A.S., MCHUGH, K.E., STEPHENS, J.A., LI, Z., et al., The Microcystic, Elongated, and Fragmented (MELF) Pattern of Invasion: A Single Institution Report of 464 Consecutive FIGO Grade 1 Endometrial Endometrioid Adenocarcinomas, The American Journal of Surgical Pathology, 41(1), 2017, 49-55.

14. *** https://www.nccn.org/ 
15. BROOKS, R.A., et al., Current recommendations and recent progress in endometrial cancer, $C A$ Cancer J Clin, 69, 2019, 258-279.

16. KOMMOS, S., et al., Final validation of the ProMisE molecular classifier for endometrial carcinoma in a large population-based case series, Annals of Oncology, 29(5), 2018, 1180 - 1188.

17. GRUSHKO, T. A., et al., An exploratory analysis of HER-2 amplification and overexpression in advanced endometrial carcinoma: A Gynecologic Oncology Group study, Gynecologic oncology 108(1), 2008, 3-9.

18. WORTMAN, B.G., NOUT, R.A., BOSSE, T., Selecting adjuvant treatment for endometrial carcinoma using molecular risk factors, Current Oncology Reports, 21, 2019, 83.

19. McALPINE, J., LEON-CASTILLO, A., BOSSE, T., The rise of novel classification for endometrial carcinoma; integration of molecular subclasses, J Pathol, 244, 2018, 538-549.

20. KAUR, K.K., ALLAHBADIA, G., SINGH, M., An update on the classification, diagnosis and treatment of endometrial carcinoma - A systematic review, Acta Scientific Cancer Biology, 3(9), 2019, 29-37.

21. TALHOUK, A., McALPINE., J.N., New classification of endometrial cancers: the development and potential applications of genomic-based classification in research and clinical care, Gynecologic Oncology Research and Practice, 3, 2016, 14.

22. BRITTON, H., et al., Molecular classification defines outcomes and opportunities in young women with endometrial carcinoma, Gynecologic Oncology, 2019, 153(3), 487 - 495.

23. BOKHMAN, J.V., Two pathogenetic types of endometrial carcinoma, Gynecol Oncol., 15(1), 1983, $10-7$.

24. PIULATS, J.M., GUERRA, E., GIL-MARTIN, M., et al., Molecular approaches for classifying endometrial carcinoma, Gynecologic Oncology, 145(1), 2017, 200-207.

$\overline{\text { Manuscript received: } 27.03 .2020}$ 